\title{
Use of fine needle aspiration cytology on thyroid lumps
}

\author{
TAravinthan, ASK Banagala, KJPK Gamage \\ North Colombo Teaching Hospital, Ragama
}

\begin{abstract}
Introduction: Fine needle aspiration cytology (FNAC) plays a crucial role in the diagnosis of thyroid nodules and help to avoid number of surgical operations. Theoretically, FNAC should be carried out on all nodules, though currently only those displaying certain characteristics are biopsied. Indeed, performing FNAC on all nodules may be regarded as an excess of zeal. Therefore, it seems advisable that the endocrinologists should be able to confirm on the spot the necessity and utility of FNAC.
\end{abstract}

Methods: To determine the value of FNAC in the diagnosis of thyroid nodules, thyroid cytology of 110 patients with definitive histology after surgery was analysed from 2005 January to2006 December. FNAC was correlated with histology and the sensitivity and specificity were calculated. The method of FNAC and number of non-diagnostic aspirates were noted.

\begin{abstract}
Introduction: Fine needle aspiration cytology (FNAC) plays a crucial role in the diagnosis of thyroid nodules and help to avoid number of surgical operations. Theoretically, FNAC should be carried out on all nodules, though currently only those displaying certain characteristics are biopsied. Indeed, performing FNAC on all nodules may be regarded as an excess of zeal. Therefore, it seems advisable that the endocrinologists should be able to confirm on the spot the necessity and utility of FNAC.
\end{abstract}

Methods: To determine the value of FNAC in the diagnosis of thyroid nodules, thyroid cytology of 110 patients with definitive histology after surgery was analysed from 2005 January to2006 December. FNAC was correlated with histology and the sensitivity and specificity were calculated. The method of FNAC and number of non-diagnostic aspirates were noted.
Results: The overall sensitivity of FNAC detecting thyroid neoplasia was $80.2 \%$ and specificity was $97.2 \%$.

Conclusions: FNAC should be undertaken with ultrasound guidance and if possible with a pathologist in attendance to assess sample adequacy. If solitary nodules are to be observed, repeat FNAC should be undertaken because of the high false negative rate.

\section{Introduction}

Thyroid cancer accounts for only $0.4 \%$ of all cancer deaths and approximately 5 deaths per million population in the United States each year [1]. However, its clinical importance is disproportionate to its incidence because cancers of the thyroid must be differentiated from the much more frequent benign nodules (adenomas and multinodular goiters). Clinically detectable thyroid nodules occur in up to $4 \%$ of the population. With ultrasound, nodules may be found in up to $50 \%$ of the population over 60 years of age [2].

Fine needle aspiration cytology (FNAC) plays a crucial role in the diagnosis of thyroid nodules and enables the number of surgical operations to be reduced. Theoretically, FNAC should be carried out on all nodules, though currently only those displaying certain characteristics are biopsied. Therefore, it seems advisable that the endocrinologist should be able to confirm on the spot the necessity and utility of FNAC. Together with clinical and biochemical evaluation, ultrasound guided FNAC remains the first line diagnostic test in the management of thyroid nodules [3]. 


\section{Methods}

This study was performed at North Colombo Teaching Hospital (NCTH). All patients undergoing thyroid surgery from January 2005 to December 2006 at NCTH were identified and the definitive histological finding recorded along with FNAC report. The FNAC was performed by a technician under supervision of a consultant pathologist and slices examined by cytopathologist. FNAC findings were categorised as malignant cytology, suspicious cytology (follicular neoplasm), benign cytology, and inadequate sample.

\section{Results}

One hundred and ten patients (72 females, 38 males) with mean age of 46 years (range 26-59 years) underwent total thyroidectomy after FNAC. Out of one hundred and ten patients, FNAC report came as malignancy in ten patients (9.1\%). Among these 10 patients, histology proved malignancy in 9 patients. Histology revealed malignancy in fifteen out of sixteen who had suspicious FNAC. Among the seventy eight patients who had benign FNAC, there were six patients with a malignant histology. In six patients FNAC came as an inadequate sample. The malignant cytology and suspicious cytology was considered as thyroid neoplasia and specificity and sensitivity were calculated. The overall sensitivity of FNAC detecting thyroid neoplasia was $80.2 \%$ and specificity was $97.2 \%$.

\section{Discussion}

This study shows that together with clinical history and biochemical evaluation, FNAC remains a first line diagnostic test in the management of thyroid nodules. Fine needle aspiration cytologic (FNAC) examination has been widely adopted after numerous favorable reports of its accuracy [2]. The procedure is technically simple and acceptable to patients, but it requires an experienced operator and collaboration with a skilled cytopathologist who is capable of interpreting thyroid aspirations. Significant complications such as bleeding, infection, necrosis, or cyst formation are rare. Adequate specimens can be obtained in more than $90 \%$ of patients when two or three passes are prepared for analysis. When FNAC is used in experienced hands, false-negative and falsepositive diagnoses occur in less than $5 \%$ of cases. Currently, FNAC is viewed as the "gold standard" for diagnosis in most cases, and it plays a crucial role in the selection of patients for operation. Gharib and co-workers recently analysed data on 10,000 FNAC and found the procedure to be the preferred first step in the diagnosis. The diagnostic accuracy was nearly $98 \%$, with less than $2 \%$ false-positive and falsenegative results [4].

In general, $5 \%$ to $8 \%$ of aspirates are diagnostic of malignancy, $10 \%$ to $20 \%$ are considered suspicious but not diagnostic (demonstrating micro follicular cytology), $2 \%$ to $5 \%$ fail to provide an adequate specimen, and the remainder is considered benign, usually suggestive of a "colloid nodule" or thyroiditis [4].

An inadequate specimen should lead to reaspiration. A biopsy of nonpalpable nodules can be performed under ultrasound guidance. Nonpalpable thyroid nodules, typically less than $1 \mathrm{~cm}$ in size, are usually non malignant. Ultrasound-guided fine needle aspiration biopsy may be appropriate in these individuals. A positive diagnosis of cancer leads to surgery. Patients with suspicious FNAC report should also undergo surgery since approximately $25 \%$ prove to be malignant.

In one study by Sidoti $\mathrm{M}$ et al. the FNAC was performed under ultrasound guidance in accordance with a standard technique. Authors concluded that together with clinical and biochemical evaluation, ultrasound guided FNAC remains the first line diagnostic test in the management of thyroid nodules [3]. In another study done by Morgan J L et al it was concluded that FNAC was essential in the management of thyroid nodules [5]. 'Malignant' or 'suspicious for malignancy' cytology are absolute indicators for thyroidectomy. FNAC should be undertaken with ultrasound guidance and if possible with a pathologist in attendance to assess sample adequacy [6]. Recent studies conducted by 
Valiyaparambath $\mathrm{XJ}$ et al, showed that ultrasound-guided FNAC is the gold standard diagnostic tool for management of thyroid nodules [6]. According to above studies, FNAC is useful in evaluating thyroid nodules, but the non diagnostic rate of FNAC must be minimised. There are strategies to enable this, depending on local resources. Increase access to cytologists or cytology technicians, diagnostic ultrasound image guidance for FNAC and the use of ultrasound guided core biopsy [7].

\section{References}

1. Landis SH, Murray $\mathrm{T}$, Bolden $\mathrm{S}$, et al. Cancer statistics, 1999. CA Cancer Journal Clin . 1999; 49: 831 .

2. Erdogan MF, Kamel N, Aras D, et al. Value of reaspirations in benign nodular thyroid disease. Thyroid. 1998; 8: 1087-90.
3. Thomas V, Mc Caffrey, Evaluation of thyroid nodule cancer control 2000; 7(3): 223-8.

4. Gharib H, Goellner JR, Johnson DA. Fine-needle aspiration cytology of the thyroid: a 12-year experience with 11,000 biopsies. Clin Lab Med. 1993;13: 699-709.

5. Morgan J L, Serpell JW, FNAC of Thyroid nodules, ANZ Journal of Surgery 2003; 73: 480.

6. X. J. Cai, N. Valiyaparambath, P. Nixon, A. Waghorn, T. Giles and T. Helliwell. (2006) Ultrasound-guided fine needle aspiration cytology in the diagnosis and management of thyroid nodules. Cytopathology 17:5, 251-6.

7. Helen H. Wang. (2006) Reporting thyroid fine-needle aspiration: Literature review and a proposal. Diagnostic Cytopathology 34: 1, 67. 